\title{
Perfil biopsicosocial del paciente con tuberculosis y factores asociados a la adherencia terapéutica ${ }^{+}$
}

\author{
PÍA MOLINA-CHAILÁN*, SARA MENDOZA-PARRA**, \\ KATIA SÁEZ C. ${ }^{* * *}$ y SALVADOR CABRERA F.****,*****
}

\begin{abstract}
Biopsychosocial profile of the patient with tuberculosis and factors associated with therapeutic adherence
\end{abstract}

Background: Antibiotic resistance and inadequate therapeutic adherence are phenomena that promote the proliferation of tuberculosis. Sociodemographic changes challenge us to know the real situation of the disease and allows us to contextualize a new scenario. Objective: To characterize the biopsychosocial profile of the patient with tuberculosis and its relationship to therapeutic adherence. Material and Method: Descriptive, cross-sectional, correlational study. Sample of 90 patients treated at 35 Family Health Centers from the following Chilean Public Health Services: Iquique, Metropolitan northern (Santiago), Concepción and Reloncaví. Results: Biopsychosocial components such as age, history of mental illness, self-esteem, sentimental status, belonging to risk groups, alcoholism, drug addiction and homeless situation presented a statistically significant relationship with therapeutic adherence. Conclusions: The biopsychosocial characterization of the TB patient evidence a new adherence-related factors that should be considered for interdisciplinary care.

Key words: Tuberculosis; Treatment adherence and compliance, Cross-sectional studies; Alcoholism; homeless persons; Drug resistance. Microbial, Self concept.

\section{Resumen}

Introducción: La resistencia antibiótica y una inadecuada adherencia terapéutica son fenómenos que favorecen la proliferación de la tuberculosis. Los cambios sociodemográficos nos desafían a conocer la realidad actual de la enfermedad a través de antecedentes que nos permitan contextualizar un nuevo escenario. Objetivo: Caracterizar el perfil biopsicosocial del paciente con tuberculosis y su relación con la adherencia terapéutica. Material y Método: Estudio descriptivo, transversal, correlacional. Muestra de 90 pacientes tratados en 35 Centros de Salud Familiar de los Servicios de Salud de Iquique, Metropolitano Norte, Concepción y Reloncaví. Resultados: los componentes biopsicosociales como edad, antecedentes de enfermedad mental, autoestima, situación sentimental, pertenencia a grupos de riesgo, alcoholismo, drogadicción y situación de calle presentaron una relación estadísticamente significación con la adherencia terapéutica. Conclusiones: La caracterización biopsicosocial del paciente con tuberculosis visibiliza nuevos factores relacionados con la adherencia que deben ser considerados para una atención interdisciplinaria.

Palabras clave: Tuberculosis; Adherencia Terapéutica; Estudios descriptivos transversales; Alcoholismo; personas en situación de calle; Resistencia microbiana a drogas; Autoestima.

\footnotetext{
+Trabajo financiado por: Beca Doctorado Nacional folio 21160745, Comisión Nacional de Investigación Científica y Tecnológica (CONICYT). Proyecto VRID-multidisciplinario código 219.202.002-M, Vicerrectoría de Investigación y Desarrollo, Universidad de Concepción.

* Candidata grado Doctor en Enfermería, Universidad de Concepción. Concepción, Chile.

** Doctor en Enfermería, Departamento Fundamentos de Enfermería y Salud Pública, Facultad de Enfermería, Universidad de Concepción. Concepción, Chile.

*** Doctor en Ingeniería Eléctrica, Magister en Estadística, Departamento de Estadística, Facultad Ciencias Físicas y Matemáticas, Universidad de Concepción. Concepción, Chile.

**** Doctor en Farmacocinética Experimental y Clínica, Departamento de Farmacia, Facultad de Farmacia, Universidad de Concepción. Concepción, Chile.

***** Unidad de Terapias Personalizadas, Hospital Clínico Regional Dr. Guillermo Grant Benavente. Concepción, Chile.
} 


\section{Introducción}

La tuberculosis es una enfermedad transmisible que se ubica dentro de las 10 primeras causas de muerte en el mundo y es la principal causa de muerte por un solo agente infeccioso ${ }^{1}$. El año 2018 marcó un hito para el manejo y control de la enfermedad, ya que se realizó la primera reunión de alto nivel de la Asamblea General de las Naciones Unidas sobre 'el Fin de la tuberculosis'. En esta asamblea se celebró el compromiso internacional de reducir en un $90 \%$ las muertes y el $80 \%$ la tasa de incidencia para el año $2030^{1}$.

La Región de las Américas representa el 3\% de la carga mundial de la enfermedad, registrando una tasa de incidencia de 46,2 casos por $100 \mathrm{mil}$ habitantes en la subregión América del Sur para el año 2017 , donde el $45 \%$ de los casos se presentan en Brasil y Perú ${ }^{2}$.

Chile, por su parte, tiene una de las tasas de incidencia de tuberculosis más bajas de la región, habiéndose planteado su eliminación como problema de salud pública para el año 2020, es decir, alcanzar una tasa de incidencia menor a 5 casos por 100 mil habitantes. Sin embargo, en los últimos años la tasa de incidencia y morbilidad ha ido en aumento pasando de una tasa de 13,4 el año 2014 a 15,7 casos por 100 mil habitantes para el año el $2018^{3,4}$.

Si deseamos reducir las fuentes de trasmisión social de la tuberculosis debemos asegurar la curación fundamentalmente de los casos pulmonares sintomáticos respiratorios bacteriológicamente demostrados. Sin embargo, los resultados de los tratamientos de estos pacientes indican que existe una proporción importante de abandonos a la terapia. La OMS recomienda mantener una proporción de abandonos no mayor de 5\% para todos los tipos de tuberculosis. En Chile, la cohorte de casos nuevos del año 2017 registran $6,8 \%$ de pérdida de seguimiento pero hay $7 \mathrm{ser}$ vicios de salud con cifras superiores a $10 \%$ de abandono. Para los casos previamente tratados la situación es peor en la cohorte de 2017 ya que registran $26,1 \%$ de abandono ${ }^{5}$.

Uno de los objetivos del Programa de Control y Eliminación de la Tuberculosis (PROCET) es lograr un $90 \%$ de curación de los casos de tuberculosis, y dentro de las estrategias relacionadas a este objetivo se contempla la prevención de la pérdida de seguimiento de los pacientes (antiguamente llamado 'abandono') ${ }^{6}$. La aplicación del puntaje de riesgo de abandono en la primera consulta de enfermería es una de las medidas preventivas utilizadas para esto, ya que según su puntuación es posible anticipar el riesgo de abandono ${ }^{6,7}$. Creado el año 2005, estableció que las personas con antecedentes de alcoholismo, drogadicción, sin previsión, que viven solos y que tienen antecedentes de abandonos previos tenían mayor riesgo de abandonar ${ }^{8}$. Sin embargo, el año 2013, a través de las auditorias de los casos de abandono, se detectó que las personas en situación de calle, coinfectadas con VIH y con patologías psiquiátricas también abandonaban el tratamiento ${ }^{7}$.

A esto se suma el hecho de que la tuberculosis se ha ido concentrando en los últimos años en los grupos vulnerables, entre los cuales se encuentran personas que presentan las mismas comorbilidades o perfiles relacionados con el riesgo de abandono: alcoholismo, drogadicción, personas en situación de calle y personas viviendo con $\mathrm{VIH}^{9}$. Este panorama advierte un cambio sociodemográfico de las personas que padecen de tuberculosis y su comportamiento adherente frente al tratamiento. Así, componentes psicosociales, tales como enfermedades mentales y/o condiciones de vida, comienzan a irrumpir con fuerza como probables causales de no adherencia al tratamiento en estos pacientes ${ }^{10}$.

El enfoque biopsicosocial de la salud considera al individuo no sólo como un organismo biológico, sino como una persona que posee una estructura psicológica compleja, en interacción constante con otros organismos, en un entorno social diverso. Este enfoque permite comprender el estado de salud- enfermedad como un proceso dinámico producto de la interacción de factores biológicos, psicológicos y sociales, incorporando al paciente como sujeto y no objeto del proceso asistencial. De esta forma, la práctica del modelo genera una comunicación que nace del supuesto que la realidad de cada persona se interpreta a través del diálogo entre el equipo de salud, su entorno y la persona ${ }^{11,12}$. Esta relación de salud logra resultados terapéuticos como la continuidad del tratamiento y mejores pronósticos de la enfermedad ${ }^{11}$.

Por todo lo expuesto, surge la necesidad de contar con un perfil actualizado de las personas diagnosticadas con tuberculosis, por lo que este estudio tiene como objetivo caracterizar el perfil biopsicosocial del paciente con tuberculosis y su relación con la adherencia terapéutica.

\section{Materiales y Métodos}

\section{Tipo de estudio y muestra}

Estudio de abordaje cuantitativo, descriptivo, transversal, correlacional. Los Servicios de Salud 
(SS) fueron seleccionados conjuntamente entre los investigadores y los encargados de PROCET a nivel ministerial, considerando representatividad de Servicios de Salud del norte, centro y sur del país. Así, entre los meses de septiembre de 2018 y junio de 2019 de un total 42 Centros de Salud Familiar (CESFAM) de los Servicios de Salud Iquique, Metropolitano Norte, Concepción y Reloncaví, 35 CESFAM aceptaron participar en esta investigación. Los criterios de inclusión definidos fueron: pacientes con 15 años o más, en tratamiento por tuberculosis en un CESFAM perteneciente al área de jurisdicción. Los criterios de exclusión: pacientes con deterioro físico y/o cognitivo severo que impida responder al instrumento diseñado y no pueda ser representado por su cuidador, y pacientes en tratamiento por tuberculosis hospitalizados al momento de la entrevista.

\section{Resguardos éticos}

Esta investigación respetó las normas éticas concordantes con la Declaración de Helsinki y fue autorizado por los Comités Ético Científicos de la Facultad de Enfermería y la Vicerrectoría de Investigación y Desarrollo de la Universidad de Concepción, Servicio de Salud Metropolitano Norte, Servicio de Salud Concepción, Corporación Municipal de Desarrollo Social de Iquique y Servicio de Salud Reloncaví.

Se solicitó consentimiento y/o asentimiento informado previo a la entrevista con los participantes.

\section{Instrumentos}

Se utilizaron un total de seis instrumentos, que se describen brevemente a continuación:

1) Cuestionario sociodemográfico confeccionado por los autores, el cual consta de 35 preguntas que recogen información sociodemográfica y clínica del paciente, a través de preguntas abiertas y datos obtenidos de ficha clínica (tarjetón de tratamiento).

2) Recuento de comprimidos (pill count) ${ }^{13,14}$ : establece el porcentaje de cumplimiento a través del cociente obtenido entre la dosis diaria ingerida y los días que debió ingerir.

3) Cuestionario Apoyo Social MOS (The Medical Outcomes Study Social Support Survey) $)^{15,16}$ con 19 ítems que evalúan el apoyo social global, con un puntaje que fluctúa entre 19 a 95 puntos, a mayor puntaje mayor apoyo social. Incluye ítem para evaluar apoyo estructural, que midió el número de amigos y familiares significativos para el participante.
4) Escala de evaluación de la cohesión y flexibilidad familiar (FACES III) ${ }^{17}$ con 20 ítems que miden cohesión, definida como el lazo emocional existente entre los miembros de la familia y flexibilidad, que es la capacidad de cambiar el liderazgo o roles familiares frente al estrés. Los niveles de cohesión se clasificaron en: desvinculado (10-34 puntos), separado (35 a 40 puntos), relacionado (41-45 puntos) y aglutinado (46-50 puntos). Los niveles de flexibilidad se clasificaron en: rígido (10-19 puntos), estructurado (20-24 puntos), flexible (25-29 puntos) y caótico (30-50 puntos).

5) Escala de autoeficacia general ${ }^{18}$, de 10 ítems con 4 opciones de respuesta tipo Likert que mide la conducta de salud personal, con un puntaje que fluctúa entre 10 y 40 puntos, a mayor puntaje mayor autoeficacia.

6) Escala de autoestima de Rosenberg ${ }^{19,20}$, de 10 ítems con 4 opciones de respuesta tipo Likert, que mide los sentimientos de respeto y aceptación de sí mismo, con un puntaje total de 40 puntos, a mayor puntaje mayor autoestima.

\section{Control de calidad de los datos}

Con objeto de evaluar el grado de comprensión de los instrumentos, se llevó a cabo una prueba piloto en 30 personas tratadas por patologías respiratorias, incluida tuberculosis, en el policlínico de Broncopulmonar del Hospital Clínico Dr. Guillermo Grant Benavente, Concepción. Posterior a la aplicación de los instrumentos, y para observar las características psicométricas de las escalas, se obtuvo la confiabilidad interna mediante Alpha de Cronbach (Tabla 1).

El instrumento en su totalidad fue aplicado en formato de entrevista por tres enfermeros con formación en investigación y experiencia en atención primaria. Se confeccionó un "Manual del Encuestador" y se realizó una capacitación previa al inicio de la recolección de datos.

Tabla 1. Confiabilidad de los instrumentos utilizados en pacientes con tuberculosis $(n=90)$

\begin{tabular}{|lc|}
\hline Escalas & $\begin{array}{c}\text { Alpha de } \\
\text { Cronbach }\end{array}$ \\
\hline $\begin{array}{l}\text { Cuestionario Apoyo Social MOS } \\
\begin{array}{l}\text { Escala de evaluación de la cohesión } \\
\text { familiar (FACES III) }\end{array}\end{array}$ & 0,96 \\
\hline $\begin{array}{l}\text { Escala de evaluación de la flexibilidad } \\
\text { familiar (FACES III) }\end{array}$ & 0,94 \\
$\begin{array}{l}\text { Escala de autoeficacia general } \\
\text { Escala de autoestima de Rosenberg }\end{array}$ & 0,83 \\
\hline
\end{tabular}




\section{Análisis estadístico}

La variable respuesta fue adherencia terapéutica, medida a través del recuento de comprimidos y cuyo análisis descriptivo consideró dos grupos: adherentes, cuando el porcentaje de cumplimiento fue $\geq 80 \%$; y no adherentes, cuando el porcentaje de cumplimiento fue $<80 \%$. Según la naturaleza de las variables explicativas se utilizaron tablas de frecuencia, medidas de tendencia central. Para el análisis inferencial bivariado, la variable adherencia al tratamiento fue el porcentaje total de cumplimiento obtenido en el conteo de píldoras, utilizando las pruebas estadísticas ' $U$ ' de Mann-Whitney, Kruskall- Wallis y Correlación de Spearman, con una significancia estadística de $\alpha=0,05$. Se utilizó el software SPSS versión 24 (Statistical Package for the Social Science).

\section{Resultados}

De un total de 209 personas con TBC en tratamiento, en los 35 CESFAM colaboradores, la muestra fue de 90 pacientes (43\%). El grupo tuvo un cumplimiento medio de $90,7 \%$ en la ingesta de fármacos antituberculosos, sustentado, mayoritariamente, por 77 adherentes $(85,6 \%)$ y de los 13 no adherentes $(14,4 \%), 5$ pertenecían al Servicio de Salud Concepción y 4 a los SS Iquique y Metropolitano Norte, respectivamente.

\section{Perfil el de las personas con tuberculosis según componentes biosociodemográficos}

\section{Componentes biológicos (Tabla 2)}

Entre los participantes, $52(57,8 \%)$ eran hombres, con un promedio de 44,5 años y $73(81,1 \%)$ tenía diagnóstico de TBC Pulmonar. El análisis de datos de la ficha clínica permitió conocer que el 52,2\% presentaba entre una o más patologías (comorbilidad), de estas, las tres más frecuentes registradas fueron alcoholismo $14 \%(\mathrm{n}=13)$, drogadicción $14 \%(\mathrm{n}=13)$ y diabetes mellitus $12 \%(n=11)$. La media de los días transcurridos entre el inicio de los síntomas y el diagnóstico de tuberculosis, para todo el grupo, fue de 190,7 días, que en el $87,8 \%$ se trataba de nuevos casos, cuyos signos y síntomas iniciales, descritos por los participantes en la entrevista, fueron: tos $(77 \% ; n=69)$, pérdida de peso $(66 \% ; n=59)$ y sudoración $(61 \% ; n=55)$, con similar tendencia entre el grupo de adherentes y los no adherentes. La media de peso de los participantes fue de 58,9 $\mathrm{kg}$, observándose un peso inferior en el grupo no adherentes de $52,7 \mathrm{~kg}$.

Tabla 2. Componentes biológicos y adherencia terapéutica en pacientes tratados por tuberculosis $(\mathbf{n}=90)$

\begin{tabular}{|c|c|c|c|c|}
\hline Variable & $\begin{array}{c}\text { Adhiere } \\
\text { n (\%) }\end{array}$ & $\begin{array}{c}\text { No adhiere } \\
\text { n (\%) }\end{array}$ & $\begin{array}{l}\text { Total } \\
\text { n (\%) }\end{array}$ & $\mathbf{p}$ \\
\hline $\begin{array}{l}\text { Sexo } \\
\text { Hombre } \\
\text { Mujer } \\
\text { Total }\end{array}$ & $\begin{array}{lr}43 & (55,8) \\
34 & (44,2) \\
77 & (100,0)\end{array}$ & $\begin{array}{rr}9 & (69,2) \\
4 & (30,8) \\
13 & (100,0)\end{array}$ & $\begin{array}{lr}52 & (57,8) \\
38 & (42,2) \\
90 & (100,0)\end{array}$ & 0,422 \\
\hline $\begin{array}{l}\text { Localización Tuberculosis } \\
\text { Pulmonar } \\
\text { Extrapulmonar } \\
\text { Total }\end{array}$ & $\begin{array}{ll}62 & (80,5) \\
15 & (19,5) \\
77 & (100)\end{array}$ & $\begin{aligned} 11 & (84,6) \\
2 & (15,4) \\
13 & (100)\end{aligned}$ & $\begin{array}{ll}73 & (81,1) \\
17 & (18,9) \\
90 & (100)\end{array}$ & 0,473 \\
\hline $\begin{array}{l}\text { Tipo de caso } \\
\text { Nuevo } \\
\text { Recaída/Reingreso por abandono } \\
\text { Total }\end{array}$ & $\begin{aligned} 69 & (89,6) \\
8 & (10,4) \\
77 & (100)\end{aligned}$ & $\begin{aligned} 10 & (76,9) \\
3 & (23,1) \\
13 & (100)\end{aligned}$ & $\begin{array}{ll}79 & (87,8) \\
11 & (12,2) \\
90 & (100)\end{array}$ & 0,134 \\
\hline $\begin{array}{l}\text { Comorbilidades } \\
\text { No } \\
\mathrm{Si} \\
1 \text { o } 2 \text { patologías } \\
3 \text { o más patologías } \\
\text { Total }\end{array}$ & $\begin{array}{c}38(49,3) \\
39(50,7) \\
34(44,2) \\
5(6,5) \\
77(100)\end{array}$ & $\begin{array}{rr}5 & (38,4) \\
8 & (61,6) \\
4 & (30,8) \\
4 & (30,8) \\
13 & (100)\end{array}$ & $\begin{aligned} 43 & (47,8) \\
47 & (52,2) \\
38 & (42,2) \\
9 & (10,0) \\
90 & (100)\end{aligned}$ & 0,118 \\
\hline Edad Media (DS) & $44,9(19,7)$ & $41,8(21,6)$ & $44,5(19,9)$ & 0,038 \\
\hline Tiempo transcurrido $^{(1)} \quad$ Media (DS) & $190,7(272,8)$ & $190,8(165,7)$ & $190,7(261)$ & 0,238 \\
\hline Peso $^{(2)}$ Media (DS) & $59,8(11,9)$ & $52,5(7)$ & $58,9(11,6)$ & 0,108 \\
\hline
\end{tabular}

${ }^{(1)}$ Días transcurridos entre el inicio de signos- síntomas y el diagnóstico de tuberculosis. ${ }^{(2)}$ Peso en kilogramos. 


\section{Componentes psicológicos (Tabla 3)}

La prevalencia de enfermedad mental en el grupo fue de $6,7 \%$, representado por 6 pacientes, y de ellos, 2 estaban en tratamiento por depresión, 3 con sospecha y uno con trastorno de personalidad en tratamiento. Las personas en tratamiento por depresión integraban el grupo de adherentes, mientras que los casos sospechosos o con trastorno de personalidad resultaron no adherentes. del total de participantes, la autoestima

Tabla 3. Componentes psicológicos y adherencia terapéutica en pacientes tratados por tuberculosis $(n=90)$

\begin{tabular}{|c|c|c|c|c|c|}
\hline Variable & & $\begin{array}{c}\text { Adhiere } \\
\text { n (\%) }\end{array}$ & $\begin{array}{c}\text { No adhiere } \\
\text { n (\%) }\end{array}$ & $\begin{array}{l}\text { Total } \\
\text { n (\%) }\end{array}$ & $\mathbf{p}$ \\
\hline $\begin{array}{l}\text { Enfermedades mentale } \\
\text { Sí } \\
\text { No } \\
\text { Total }\end{array}$ & & $\begin{array}{rr}2 & (2,6) \\
75 & (97,4) \\
77 & (100)\end{array}$ & $\begin{aligned} 4 & (30,8) \\
9 & (69,2) \\
13 & (100)\end{aligned}$ & $\begin{array}{rr}6 & (6,7) \\
84 & (93,3) \\
90 & (100)\end{array}$ & $0,006^{*}$ \\
\hline Autoestima & Media (DS) & $30,5(5,7)$ & $27,7(6,6)$ & $30,1(5,8)$ & $0,031^{*}$ \\
\hline Autoeficacia general & Media (DS) & $33,2(5,9)$ & $30,2(5,3)$ & $32,8(5,9)$ & 0,107 \\
\hline
\end{tabular}

Tabla 4. Componentes sociales y adherencia terapéutica en pacientes tratados por tuberculosis $(\mathbf{n}=90)$

\begin{tabular}{|c|c|c|c|c|c|}
\hline Variable & & $\begin{array}{c}\text { Adhiere } \\
\text { n (\%) }\end{array}$ & $\begin{array}{c}\text { No adhiere } \\
\text { n (\%) }\end{array}$ & $\begin{array}{l}\text { Total } \\
\text { n (\%) }\end{array}$ & $\mathbf{p}$ \\
\hline $\begin{array}{l}\text { Vivienda }(\mathrm{n}=87) \\
\text { Casa/departamento } \\
\text { Pieza } \\
\text { Total }\end{array}$ & & $\begin{array}{ll}50 & (65,8) \\
26 & (34,2) \\
76 & (100)\end{array}$ & $\begin{array}{c}9(81,8) \\
2(18.2) \\
11(100)\end{array}$ & $\begin{array}{ll}59 & (67,8) \\
28 & (32,2) \\
87 & (100)\end{array}$ & 0,242 \\
\hline $\begin{array}{l}\text { Nivel educacional } \\
\text { Básica completa } \\
\text { Básica incompleta } \\
\text { Media completa } \\
\text { Media incompleta } \\
\text { Superior } \\
\text { Total }\end{array}$ & & $\begin{aligned} 13 & (16,9) \\
9 & (11,7) \\
27 & (35) \\
13 & (16,9) \\
15 & (19,5) \\
77 & (100)\end{aligned}$ & $\begin{aligned} & 1(7,7) \\
5 & (38,5) \\
2 & (15,4) \\
2 & (15,4) \\
3 & (23) \\
13 & (100)\end{aligned}$ & $\begin{array}{ll}14 & (15,6) \\
14 & (15,6) \\
29 & (32,2) \\
15 & (16,6) \\
18 & (20) \\
90 & (100)\end{array}$ & 0,098 \\
\hline $\begin{array}{l}\text { Situación sentimental } \\
\text { Con pareja } \\
\text { Sin pareja } \\
\text { Total }\end{array}$ & & $\begin{array}{ll}40 & (52) \\
37 & (48) \\
77 & (100)\end{array}$ & $\begin{aligned} 4 & (30,8) \\
9 & (69,2) \\
13 & (100)\end{aligned}$ & $\begin{array}{ll}44 & (48,9) \\
46 & (51,1) \\
90 & (100)\end{array}$ & 0,029 \\
\hline $\begin{array}{l}\text { Cohesión familiar } \\
\text { Aglutinada } \\
\text { Relacionada } \\
\text { Separada } \\
\text { Desvinculada } \\
\text { Total }\end{array}$ & & $\begin{array}{ll}24 & (31,2) \\
16 & (20,8) \\
16 & (20,8) \\
21 & (27,2) \\
77 & (100)\end{array}$ & $\begin{aligned} 4 & (30,8) \\
0 & (0) \\
4 & (30,8) \\
5 & (38,4) \\
13 & (100)\end{aligned}$ & $\begin{array}{ll}28 & (31,1) \\
16 & (17,8) \\
20 & (22,2) \\
26 & (28,9) \\
90 & (100)\end{array}$ & 0,097 \\
\hline $\begin{array}{l}\text { Flexibilidad Familiar } \\
\text { Caótica } \\
\text { Flexible } \\
\text { Estructurada } \\
\text { Rígida } \\
\text { Total }\end{array}$ & & $\begin{array}{ll}34 & (44,1) \\
13 & (16,9) \\
12 & (15,6) \\
18 & (23,4) \\
77 & (100)\end{array}$ & $\begin{array}{rr}7 & (53,8) \\
1 & (7,7) \\
2 & (15,4) \\
3 & (23,1) \\
13 & (100)\end{array}$ & $\begin{array}{l}41(45,5) \\
14(15,6) \\
14(15,6) \\
21(23,3) \\
90(100)\end{array}$ & 0,535 \\
\hline $\begin{array}{l}\text { Grupos de Riesgo } \\
1 \text { grupo } \\
2 \text { grupos } \\
3 \text { o más grupos } \\
\text { Ninguno } \\
\text { Total }\end{array}$ & & $\begin{aligned} 36 & (46,8) \\
17 & (22) \\
8 & (10,4) \\
16 & (20,8) \\
77 & (100)\end{aligned}$ & $\begin{array}{cc}7 & (53,8) \\
2 & (15,4) \\
4 & (30,8) \\
0 & (0) \\
13 & (100)\end{array}$ & $\begin{array}{ll}43 & (47,8) \\
19 & (21,1) \\
12 & (13,3) \\
16 & (17,8) \\
90 & (100)\end{array}$ & 0,009 \\
\hline Apoyo social global $(\mathrm{n}=90)$ & Media (DS) & $73,5(20,5)$ & $71,5(26,6)$ & $73,2(21,4)$ & 0,335 \\
\hline Apoyo social estructural $(n=90)$ & Media (DS) & $3,8(4,4)$ & $1,8(1,2)$ & $3,5(4,1)$ & 0,293 \\
\hline
\end{tabular}


presentó una media de 30,1 puntos de un total de 40 puntos, el grupo de no adherentes exhibió un puntaje inferior al total de 27,7 puntos. Mientras que, la autoeficacia general obtuvo un puntaje de 32,8 de un total de 40 puntos, registrándose menor puntaje en el grupo de no adherentes.

\section{Componentes sociales (Tablas 4 y 5 )}

Según tipo de vivienda, el $67,8 \%(\mathrm{n}=59)$ vive en casa o departamento, el 32,3\% $(\mathrm{n}=29)$ presentó enseñanza media completa y el $51,1 \%$ $(n=46)$ señaló al momento de la entrevista no tener pareja sentimental. La cohesión familiar se caracterizó por ser de tipo aglutinada en el 31,1\% $(\mathrm{n}=28)$, con una flexibilidad familiar de tipo caótica en el 45,5\% $(n=41)$ de los participantes. Según la pertenencia a grupos de riesgo, todos los participantes no adherentes quedaron en algún grupo de riesgo (Tabla 4). Con respecto a los extranjeros, provienen principalmente de Perú 20\% $(\mathrm{n}=18)$, Bolivia $11,1 \%(\mathrm{n}=10)$ y Haití $6,7 \%$ $(n=6)$, con una media de estadía en nuestro país de 5,9 \pm 6 años (Tabla 5).

\section{Componentes biopsicosociales y adherencia terapéutica}

De las seis variables medidas en el componente biológico, la edad presentó una correlación positiva (Rho Spearman $=0,219 ; p=0,038$ ) con la adherencia al tratamiento, es decir, a mayor edad mayor adherencia terapéutica (Tabla 2).

Respecto al componente psicológico, la presencia de enfermedades mentales (U MannWhitney $=83,500 ; p=0,006)$ y la autoestima presentaron una correlación positiva (Rho Spearman $=0,228 ; p=0,031)$ con la adherencia terapéutica, es decir, si el paciente junto a la TBC presenta una enfermedad mental tiene menores probabilidades de ser adherente y a mayor autoestima mayor adherencia terapéutica.

Respecto al componente social, existe una relación estadísticamente significativa entre la adherencia terapéutica y la situación sentimental (U Mann-Whitney $=742,500 ; p=0,029)$ y la pertenencia a grupos de riesgo (KruskallWallis $=11,676 ; p=0,009)$. De estos, el alcoho-

Tabla 5. Grupos de riesgo y adherencia terapéutica en pacientes tratados por tuberculosis

\begin{tabular}{|c|c|c|c|c|}
\hline Grupo de riesgo & $\begin{array}{l}\text { Adhiere } \\
\text { n (\%) }\end{array}$ & $\begin{array}{c}\text { No adhiere } \\
\text { n (\%) }\end{array}$ & $\begin{array}{l}\text { Total } \\
\text { n (\%) }\end{array}$ & $\mathbf{p}$ \\
\hline $\begin{array}{l}\text { VIH } \\
\text { Sí } \\
\text { No }\end{array}$ & $\begin{array}{rr}3 & (3,9) \\
74 & (96,1)\end{array}$ & $\begin{array}{r}2(15,4) \\
11(84,6)\end{array}$ & $\begin{array}{rr}5 & (5,6) \\
85 & (94,4)\end{array}$ & 0,088 \\
\hline $\begin{array}{l}\text { Extranjeros } \\
\text { Sí } \\
\text { No }\end{array}$ & $\begin{array}{l}34(44,2) \\
43(55,8)\end{array}$ & $\begin{array}{l}5(38,5) \\
8(61,5)\end{array}$ & $\begin{array}{l}39(43,3) \\
51(56,7)\end{array}$ & 0,984 \\
\hline $\begin{array}{l}\text { Alcoholismo } \\
\text { Sí } \\
\text { No }\end{array}$ & $\begin{array}{r}7(9) \\
70(91)\end{array}$ & $\begin{array}{r}3(23,1) \\
10(76,9)\end{array}$ & $\begin{array}{l}10(11,1) \\
80(88,9)\end{array}$ & 0,048 \\
\hline $\begin{array}{l}\text { Drogadicción } \\
\text { Sí } \\
\text { No }\end{array}$ & $\begin{array}{r}7(9) \\
70(91)\end{array}$ & $\begin{array}{l}5(38,5) \\
8(61,5)\end{array}$ & $\begin{array}{l}12(13,3) \\
78(86,7)\end{array}$ & 0,016 \\
\hline $\begin{array}{l}\text { Diabetes mellitus } \\
\text { Sí } \\
\text { No }\end{array}$ & $\begin{array}{r}9(11,7) \\
68(88,3)\end{array}$ & $\begin{array}{r}2(15,4) \\
11(84,6)\end{array}$ & $\begin{array}{l}11(12,2) \\
79(87,8)\end{array}$ & 0,661 \\
\hline $\begin{array}{l}\text { Situación de calle } \\
\text { Sí } \\
\text { No }\end{array}$ & $\begin{array}{rr}5 & (6,5) \\
72 & (93,5)\end{array}$ & $\begin{array}{r}2(15,4) \\
11(84,6)\end{array}$ & $\begin{array}{r}7(7,8) \\
83(92,2)\end{array}$ & 0,041 \\
\hline $\begin{array}{l}\text { Contacto } \\
\text { Sí } \\
\text { No }\end{array}$ & $\begin{array}{l}22(28,6) \\
55(71,4)\end{array}$ & $\begin{array}{l}4(30,8) \\
9(69,2)\end{array}$ & $\begin{array}{l}26(28,9) \\
64(71,1)\end{array}$ & 0,617 \\
\hline $\begin{array}{l}\text { Pueblos originarios } \\
\text { Sí } \\
\text { No }\end{array}$ & $\begin{array}{rr}7 & (9) \\
70(91)\end{array}$ & $\begin{array}{r}1(7,7) \\
12(92,3)\end{array}$ & $\begin{array}{rr}8 & (8,9) \\
82 & (91,1)\end{array}$ & 0,580 \\
\hline $\begin{array}{l}\text { Adultos mayores } \\
\text { Sí } \\
\text { No }\end{array}$ & $\begin{array}{l}14(18,1) \\
63(81,9)\end{array}$ & $\begin{array}{r}2(15,4) \\
11(84,6)\end{array}$ & $\begin{array}{l}16(17,8) \\
74(82,2)\end{array}$ & 0,070 \\
\hline
\end{tabular}


lismo (U Mann-Whitney $=246,000 ; \mathrm{p}=0,048$ ), la drogadicción (U Mann-Whitney = 265,000; $\mathrm{p}=0,016)$ y la situación de calle (U MannWhitney $=155,000 ; p=0,041)$ tienen relación estadísticamente significativa con la adherencia terapéutica.

\section{Discusión}

De acuerdo con los datos presentados es posible caracterizar biopsicosocialmente al paciente con tuberculosis. Se mantienen tendencias generales como el predominio masculino y la pertenencia a un grupo etario económicamente activo $^{1}$, de estos, los resultados obtenidos indican que, a mayor edad mayor cumplimiento a la terapia farmacológica $(\mathrm{p}=0,038)$, a pesar de que los resultados no fueron significativos en el grupo de riesgo de los adultos mayores.

Los componentes psicológicos resultaron altamente significativos en la adherencia terapéutica $(\mathrm{p}=0,006)$, los antecedentes de enfermedades mentales expuestos podrían reflejar una eventual subnotificación de los casos de depresión y, por consiguiente, interferir con la adherencia a la terapia farmacológica, cuestión relevante si se sabe que los pacientes depresivos afrontan negativamente la terapia antituberculosa favoreciendo el abandono ${ }^{21}$. Actualmente en Chile no se utilizan herramientas para pesquisar tempranamente el riesgo de abandono del tratamiento tuberculoso por enfermedades mentales, ni tampoco el grupo que las sufre son considerados como vulnerables de padecer tuberculosis, aun cuando a nivel internacional ya existen estudios que señalan que el riesgo de padecer tuberculosis es 2.63 veces mayor en pacientes con depresión, existiendo una relación lineal entre el riesgo y la severidad de la depresión ${ }^{22}$, y que es mayor el riesgo de abandono, mortalidad y menor el éxito terapéutico cuando la persona padece tuberculosis y depre$\operatorname{sión}^{23}$. En Chile, en las auditorías por abandono de tratamiento, se han detectado antecedentes de cuadros mentales, pero no necesariamente esta información es regularmente registrada en la documentación actual ${ }^{7}$.

Otro componente psicológico es la autoeficacia, los niveles presentados por los participantes fueron mayoritariamente moderados. A pesar de no observarse significancia estadística, la evidencia señala que las personas con altos niveles de autoeficacia tienen deseos de sanar y mayor confianza en terminar con éxito el tratamiento ${ }^{24}$. Respecto a la autoestima, los resultados reflejan que a mayor autoestima mayor adherencia te- rapéutica $(p=0,031)$. Feng et al (2015), señala que, durante el tratamiento, mayor autoestima contribuye a enfrentar de mejor manera el estrés producido por la enfermedad y están más receptivos a la educación sobre el tema ${ }^{25}$.

Con estos resultados, parece ser relevante incorporar una evaluación psicológica que incluya la percepción del tratamiento de los pacientes con tuberculosis si queremos anticiparnos al riesgo de no adherir a una terapia prolongada.

Respecto al componente social, la situación sentimental, la pertenencia a grupos de riesgo, principalmente antecedentes de alcoholismo, drogadicción y/o estar en situación de calle resultaron tener una relación significativa con la adherencia terapéutica. Es destacable que todos los participantes no adherentes pertenecen a algún grupo de riesgo. Hallazgos similares se han encontrado en otros países latinoamericanos, revelando que el estrato social del paciente con tuberculosis también estaría relacionado con la adherencia terapéutica ${ }^{26,27}$. Si bien se ha mejorado la pesquisa de los grupos más vulnerables de padecer tuberculosis, los hallazgos detectados podrían motivar a establecer estrategias específicas de seguimiento de estos grupos, más allá de la detección temprana de estos, ya que esto no garantiza su adherencia a la terapia prolongada. Además, es imperativo la coordinación con otros programas de salud e instituciones sociales que trabajan con estos pacientes vulnerables.

A nivel general, el apoyo social global fue moderado-alto, destacando que el apoyo estructural fue el doble en el grupo de adherentes versus los no adherentes. Si bien no existió una relación significativa con la adherencia, estos antecedentes resultan relevantes para el trabajo de los equipos de salud, ya que las personas significativas para los pacientes se transforman en oportunidades de diálogo terapéutico, favoreciendo el control y continuidad de la terapia. De acuerdo con la evidencia, el apoyo individualizado entregado por los equipos de salud debe incluir la educación, y de ser posible utilizar apoyo tecnológico ${ }^{28}$, ya que los pacientes con bajo apoyo informacional tienen 4 veces menos nivel de autoeficacia, característica importante para favorecer la adherencia ${ }^{24}$.

En nuestro estudio, las familias se caracterizaron por ser disfuncionales al igual que otras investigaciones ${ }^{29,30}$. Sin embargo, difieren en la relación de la funcionalidad familiar con la adherencia terapéutica ${ }^{31}$, ya que resultó no ser significativa. La bibliografía destaca que el $75 \%$ de los casos de no adherencia presentan disfunción familiar, incluyendo el rechazo ${ }^{32}$. Por lo contrario, familias funcionales, colaboran en la terapia a tra- 
vés del apoyo en actividades rutinarias y el sustento económico del paciente con tuberculosis ${ }^{28}$.

En la actualidad, la pesquisa de estas variables biopsicosociales no está del todo regulada a nivel ministerial y el considerarlas para orientar la atención depende más bien del interés y las competencias propias de los profesionales a cargo de los programas actuales.

Las principales limitaciones de este estudio estuvieron dadas por la dificultad en el reclutamiento de los pacientes que conformaban la muestra. Si bien se incluyeron Servicios de Salud claves para el programa a nivel nacional, las dificultades para acceder a los pacientes se presentaron por inconvenientes administrativos de los programas locales, datos inespecíficos de direcciones y números telefónicos, y en algunos casos, la negativa de participar en el estudio fue por cuestiones relacionadas con el vínculo existente entre los equipos locales con sus pacientes. En futuras investigaciones es necesario aumentar el número de participantes a objeto de lograr una mayor asociación en las variables biopsicosociales y la adherencia, pues la mayor utilidad de un perfil BPS es aportar como herramienta predictiva de la adherencia al inicio de la terapia y con ello prevenir la pérdida de seguimiento.

\section{Conclusiones}

La caracterización biopsicosocial del paciente con tuberculosis visibiliza nuevos factores relacionados con la adherencia que deben ser considerados para entregar una atención integral e interdisciplinaria. En el componente biológico, la evaluación de la adherencia por rango etario parece ser una alternativa de seguimiento válida de incorporar. En el componente psicológico, es preciso evaluar tempranamente la presencia de enfermedades mentales y el estado anímico de los pacientes, a través de la autoestima, ya que son factores importantes influyentes en la adherencia terapéutica, que actualmente no son pesquisados regularmente por los programas, la evaluación psicológica debe sobrepasar las barreras de la norma técnica y hacerse una realidad objetiva. En el componente social, la incorporación formal de la valoración del paciente y sus lazos afectivos, como el tener pareja, parece ser indispensable para la pesquisa temprana y continuidad en el tratamiento. Por otro lado, la detección de pertenecer a grupos de riesgo debe estar acompañada de estrategias específicas de control y continuidad en el tratamiento, en asociación con otros actores sociales.

Esperamos que los resultados de esta inves- tigación motiven a las autoridades a destinar recursos para que los equipos locales puedan implementar herramientas y estrategias que permitan tener una visión biopsicosocial de sus pacientes. Fortalecer la atención del programa a nivel de atención primaria es la clave para disminuir la incidencia de la enfermedad, porque sólo a nivel local se puede lograr la necesaria mirada biopsicosocial que permite observar la adherencia y prevenir el abandono del tratamiento antituberculoso.

\section{Agradecimientos}

Al Equipo PROCET MINSAL, enfermera Natalia Ruiz Lobos, enfermera Zulema Torres Gaete, Dra. Nadia Escobar Salinas y Dra. Tania Herrera Martínez. Al Equipo en terreno enfermeros Marlene Opazo Salinas, Tamara Vargas Severin y Felipe Olivares González. A colaboradores y enfermeros(as) coordinadores locales del programa de tuberculosis de los Servicios de Salud Iquique, Metropolitano Norte, Concepción y Reloncaví.

\section{Bibliografía}

1.- WORLD HEALTH ORGANIZATION. Global Tuberculosis report 2019. Ginebra, 2019. Disponible en: https://www.who.int/tb/publications/global_report/en/ [consultado el 27 de enero de 2020].

2.- ORGANIZACIÓN PANAMERICANA DE LA SALUD. Tuberculosis en las Américas 2018. Informe Regional. Washington D.C, 2018. Disponible en: https:// www.paho.org/hq/index.php?option=com_topics\&view $=$ article $\& \mathrm{id}=59 \&$ Itemid $=40776 \&$ lang $=$ es $[$ consultado el 27 de enero 2020].

3.- MINISTERIO DE SALUD. Tuberculosis: informe de situación Chile 2014. Santiago de Chile, 2015. Disponible en: https://diprece.minsal.cl [consultado el 12 de julio de 2019].

4.- MINISTERIO DE SALUD. Tuberculosis: informe de situación Chile 2017. Santiago de Chile, 2018. Disponible en: https://diprece.minsal.cl [consultado el 22 de enero de 2019].

5.- MINISTERIO DE SALUD. Evaluación nacional del Programa de control y eliminación de la tuberculosis. Registro nacional 2019 [consultado el 13 de febrero de 2020].

6.- MINISTERIO DE SALUD. Normas técnicas para el control y la eliminación de la tuberculosis. Santiago de Chile, 2014. Disponible en: https://diprece.minsal. $\mathrm{cl}$ /programas-de-salud/programas-enfermedadestransmisibles/informacion-al-profesional-tuberculosis/ [consultado el 27 de enero 2020]. 
7.- TORRES Z, HERRERA T. Perfil del paciente con tuberculosis que abandona el tratamiento en Chile. Rev Chil Enferm Respir 2015; 31: 52-7.

8.- GALIANO M, MONTESINOS N. Modelo predictivo de abandono del tratamiento antituberculoso para la Región Metropolitana de Chile. Enferm Clin 2005; 15 : 192-8.

9.- HERRERA T, LEIVA E, MARTÍN F, MIRANDA M, MORALES C. Asociación entre tuberculosis y diabetes mellitus en la Región Metropolitana. Rev Chil Enferm Respir 2013; 29: 171-5.

10.- HERRERA T. Grupos de Riesgo para tuberculosis en Chile. Rev Chil Infectol 2015; 32: 15-8.

11.- ENGEL G. The need for a new medical model: a challenge for biomedicine. Science 1977; 196 (4286): 129-36.

12.- MINISTERIO DE SALUD. En el camino a Centro de Salud Familiar. Santiago de Chile, 2008. Disponible en: http://www.bibliotecaminsal.cl/wp/wp-content/ uploads/2016/03/En-el-Camino-a-Centro-de-SaludFamiliar.pdf [consultado el 12 de enero 2019].

13.- MARQUÉZ-CONTRERAS E. Evaluación del incumplimiento en la práctica clínica. Hipertensión 2008 25: 205-13.

14.- ORUETA R, TOLEDANO P, GÓMEZ. CALCERRADA R. Cumplimiento terapéutico. Semergem 2008; 34 : 235-43.

15.- REVILLA L, LUNA J, BAILÓN E, MEDINA I. Validación del cuestionario MOS de apoyo social en atención primaria. Revista Med Fam 2005; 6: 10-8.

16.- POBLETE F, GLASINOVIC A, SAPAG J, BARTICEVIC N, ARENAS A, PADILLA O. Apoyo social y salud cardiovascular: adaptación de una escala de apoyo social en pacientes hipertensos y diabéticos en la atención primaria chilena. Aten Primaria 2015; 47: 523-31.

17.- ZEGERS B, LARRAÍN E, POLAINO-LORENTE A, TRAPP A, DÍAZ I. Validez y confiabilidad de la versión española de la escala de cohesión y adaptabilidad familiar de Olson, Russell \& Sprenke para el diagnóstico del funcionamiento familiar en la población chilena. Rev Chil Neuro-psiquiatr 2003; 41: 39-54.

18.- CID P, ORELLANA A, BARRIGA O. Validación de la escala de autoeficacia general en Chile. Rev Med Chile 2010; 138: 551-7.

19.- CONSEJERÍA DE SANITAT. Escala e instrumentos para la valoración en atención domiciliaria. Valencia, España: Generalitat valenciana, 2004. Disponible en: http://www.san.gva.es/documents/246911/251004 /V.4963-2006.pdf [consultado el 1 de marzo de 2017].

20.- ROJAS-BARAHONA C, ZEGERS B, FÖRSTER C. La escala de autoestima de Rosenberg: Validación para Chile en una muestra de jóvenes adultos, adultos y adultos mayores. Rev Med Chile 2009; 137: 791-800.

21.- SWEETLAND AC, KRITSKI A, OQUENDO MA, SUBLETTE ME, NORCINI PALA A, SILVA LR, et al. Addressing the tuberculosis-depression syndemic to end the tuberculosis epidemic. Int $\mathrm{J}$ Tuberc Lung Dis 2017; 21: 852-61.

22.- OH KH, CHOI H, KIM EJ, KIM HJ, CHO SI. Depression and risk of tuberculosis: a nationwide population-based cohort study. Int J Tuberc Lung Dis 2017; 21: 804-9.

23.- AMBAW F, MAYSTON R, HANLON C, MEDHIN $\mathrm{G}$, ALEMC A. Untreated depression and tuberculosis treatment outcomes, quality of life and disability, Ethiopia. Bull World Health Organ 2018; 96: 243-55.

24.- SOLIKHAH MM, NURSASI AY, WIARSIH W. The relationship between family's informational support and self-efficacy of pulmonary tuberculosis client. Enferm Clin 2019; S1130-8621(19).

25.- NG D, XU L. The relationship between perceived discrimination and psychological distress among Chinese pulmonary tuberculosis patients: the moderating role of self-esteem. Psychol Health Med 2015; 20: 77-185.

26.- NADJANE S, ABREU R, RIBEIRO T, TAVARES F, NUNES M, ABREU L, et al. Individual and social vulnerabilities upon acquiring tuberculosis: a literature systematic review. Int Arch Med 2014; 7: 35. Disponible en: https://www.ncbi.nlm.nih.gov/pubmed/25067955 [consultado el 7 de octubre de 2019].

27.- LÓPEZ J. Determinantes sociales de la salud en pacientes con tuberculosis - Manizales-Colombia, 2012 -2014. Archivos de Medicina (Col) 2017; 17 (1): 38-53.

28.- VAN DE BERG S, JANSEN-AALDRING N, DE VRIES G, VAN DEN HOF S. Patient support for tuberculosis patients in low-incidence countries: A systematic review. PLoS One 2018; 13 (10): e0205433.

29.- BALCÁZAR-RINCÓN L, RAMÍREZ-ALCÁNTARA Y, RODRÍGUEZ-ALONSO M. Depresión y funcionalidad familiar en pacientes con diagnóstico de tuberculosis. Rev Esp Méd Quir 2015; 20: 135-43.

30.- ROSADO-QUIAB U, CEDILLO-RIVERA R, CABRERA-GAYTAN D, VARGAS-VALERIO A. Influence of Family System Characteristics on Adherence to Directly Observed Treatment, Short-Course (Dots) in Pulmonary Tuberculosis-A Cohort Study. J Mycobac Dis 2014; 4: 1-6. Doi:10.4172/2161-1068.1000166.

31.- MARTÍNEZ-HERNÁNDEZ YO, GUZMÁN-LÓPEZ F, FLORES-PULIDO JJ, VÁZQUEZ-MARTÍNEZ $\mathrm{VH}$. Factores familiares que favorecen el apego al tratamiento en casos de tuberculosis pulmonar. Aten Fam 2014; 21: 47-9.

32.- SAMAL J. Family perspectives in the care and support of tuberculosis patients: An Indian context. J Assoc Chest Physicians 2017; 5: 67-9.

Correspondencia a:

Sara Mendoza Parra, Ph.D.

Dirección: Roosevelt 1787, Concepción.

Email: smendoza@udec.cl 